Boletín de la Sociedad Zoológica del Uruguay, 2021

Vol. 30 (1): 49-52

ISSN 2393-6940

https://journal.szu.org.uy

DOI: https://doi.org/10.26462/30.1.4

\title{
NOTA
}

\section{¿CUÁN NECESARIA ES UNA MADRE ARAÑA SUBSOCIAL PARA SU CAMADA?}

\author{
Maria de Fátima da Rocha Dias ${ }^{1}$ (D) y Carmen Viera $^{2}$ (D)
}

${ }^{1} N$ úcleo de Etologia e Evolução, Instituto de Biologia, Universidade Federal da Bahia. Brasil; ${ }^{2}$ Universidad de la República, Montevideo, Uruguay, Instituto de Investigaciones Biológicas Clemente Estable, Montevideo,

Uruguay

Autor para correspondencia: faldias@yahoo.com.br

Fecha de recepción: 21 de marzo de 2021

Fecha de aceptación: 21 de mayo de 2021

\section{RESUMEN}

El presente estudio en Anelosimus jabaquara tiene dos objetivos: registrar la posible colaboración materna en la apertura de ootecas y grado de independencia de las crías en la captura de presas. Los resultados muestran que el cuidado maternal es muy necesario en las primeras etapas del desarrollo para la supervivencia de las crías.

Palabras clave: Anelosimus, cuidado maternal, subsocialidad.

\section{ABSTRACT \\ How necessary is a subsocial spider mother for her brood? This study in Anelosimus jabaquara pursuit two objectives: to register the possible mother's collaboration on egg-sacs opening and to know the independence degree of the spiderlings in prey capture. The results show that maternal care is very necessary at the first stages of development for survival of the spiderlings.}

Keywords: Anelosimus, maternal care, subsociality

La mayoría de las arañas viven solitariamente casi toda la vida, excepto en los cortos períodos después la emergencia de la progenie (Foelix, 2011). Algunas especies de arañas extienden la fase de vida gregaria, generando grupos subsociales y sociales (Kullman, 1972). Las arañas sociales viven en telas comunales y cooperan en la captura, si bien no existen individuos especializados en funciones o castas para la defensa del territorio, la captura de presas o en la reproducción, como sí sucede en los insectos sociales (Buskirk, 1981). Cooperar en la captura y vivir en grupo necesita algunos prerrequisitos como tolerancia, interrelación y cooperación entre los individuos, fundamentalmente, la inhibición del canibalismo (Kullman, 1972). Algunas arañas han desarrollado cuidados maternos que incluyen las interacciones madre-crías, que varían en su comportamiento y grado de inversión en relación con el tipo de socialidad. Las arañas solitarias no cuidan las crías, las sociales no tienen cuidado maternal individual, sino que todas las hembras colaboran comunalmente en el cuidado de las crías, siendo las subsociales las que presentan mayor inversión individual, ya que no hay cooperación comunal (Buskirk, 1981; Aviles, 1997). Se han propuesto varias funciones para los cuidados maternales, entre ellas, la protección de las ootecas contra la depredación y/o el parasitismo (Lubin, 1974; Fink, 1986; Vieira y Romero, 2008). Asimismo, la inversión materna puede influir en la regulación de factores externos de temperatura y humedad intra-ooteca (Horel y Krafft, 1986). El cuidado de los sacos de huevos es una primera etapa de cuidado. A la vez, durante la emergencia de las arañitas se ha observado cooperación maternal y/o facilitación de la apertura de la ooteca (Ito y Shinkai, 1993; Toyama, 1999; Japyassú, Macagnan y Knysak, 2003). Esta inversión materna al inicio de la vida de la progenie puede extenderse colaborando con la provisión y abastecimiento de alimento a las crías en algunas especies (Kullmann, 1972; Buskirk, 1981). Norgaard (1956) encontró que las madres pueden además alertar al grupo sobre la presencia de peligro o alimento a los jóvenes.

La mayor parte de las arañas sociales (también denominadas permanentemente sociales) se encuentran en los trópicos (Kullman, 1972; Nava, Whitehouse y Lubin, 2000; Yip y Rayor, 2014; Avilés y Guevara, 
2017; Deshmukh, 2017) y se registran en diferentes familias: Dictynidae, Eresidae, Desidae, Agelenidae, Theridiidae y Thomisidae (Foelix, 2011). En la familia Theridiidae se encuentra el género Anelosimus que contiene arañas desde solitarias, prácticamente sin ningún cuidado parental post-eclosión, pasando por arañas subsociales con extensión del cuidado maternal (hasta subadultos) hasta arañas muy sociales donde el cuidado maternal es cooperativo en estas sociedades permanentes (Avilés, 1997; Agnarsson, 2004, 2006). Viera, Ghione y Costa (2007) han registrado que las madres intervienen activamente en el proceso de apertura de ooteca y brindan alimento a su progenie en una araña subsocial Anelosimus vierae (Agnarsson, 2012). En el presente estudio quisimos observar el comportamiento maternal en otra araña subsocial, del Brasil a efectos comparativos con $A$. vierae. Planteamos: 1) registrar la posible intervención materna en el proceso de apertura de las ootecas y 2) conocer la posible colaboración materna en la captura de presas en una araña subsocial Anelosimus jabaquara (Levi, 1956).

Se realizaron los experimentos en el laboratorio de la Base Ecológica de la Sierra do Japi, Jundiaí (23 11 'S, $46^{\circ} 52$ 'W), São Paulo, Brasil. Para ello, se utilizaron ootecas inmediatamente de haber sido puestas. Todas las arañas se mantuvieron en cajas de Petriß de $9,5 \mathrm{~cm}$ de diámetro y $1,5 \mathrm{~cm}$ de altura durante 3 meses (tiempo máximo para la eclosión) a temperatura constante de $20.4^{\circ} \mathrm{C}$. Para el primer objetivo se realizaron dos grupos: a) formado por 7 ootecas aisladas de su madre y b) 10 hembras con sus propias ootecas.

Se mantuvieron las hembras junto a sus propias crías durante 24 horas después de la eclosión para permitir la alimentación por trofalaxia, que ocurrió en todos los casos. Para analizar el segundo objetivo, se separó cada grupo con madres (b) en dos subgrupos: b1: las madres y sus crías $(n=10)$ y b2 las crías sin sus madres $(n=10)$. Al tercer día se alimentaron ambos subgrupos con la misma cantidad de Drosophila sp. y luego, cada 2 días durante una semana más. Se registraron los comportamientos de todos los lotes con observaciones de 1 hora cada vez. Todos los individuos fueron controlados y registrados a través de fichas grupales, sobre su alimentación, si comían o no y grado de desarrollo post-eclosión. Mediante el test de Shapiro-Wilk se observó que los datos no presentaron una distribución normal, utilizándose el test Wilcoxon Man-Whitney del paquete estadístico $\mathrm{R}$ versión 3.0.2. Una muestra de los ejemplares utilizados fue depositada en la colección aracnológica del Instituto Butantán (Brasil) y el resto fue liberado en los sitios de recolección.

Las ootecas presentaron una forma esférica de color marrón con un tamaño promedio de $3.57 \mathrm{~mm}$ ( $\sigma=$ $0,53)$. Cada ooteca tuvo un promedio de 34 crías $(\sigma=$ $9,09)$. El número promedio de huevos infértiles fue de $3.28(\sigma=5,34)$. Todos los individuos intra-ooteca presentaron el mismo número de mudas. Tres ootecas presentaran hongos y ácaros en su parte exterior. Después del tiempo predeterminado de desarrollo intra-ooteca, todas las ootecas fueron abiertas, porque no hubo eclosión.

Respecto a las experiencias de captura, las crías aisladas de la madre no presentaron comportamiento de captura, ya que siquiera ni respondieron a la presencia de las presas. En los grupos con presencia materna (b1), las madres capturaron y compartieron las presas con las crías. En 4 de los 5 grupos se observaron mudas en los juveniles después de los experimentos, mientras que en los grupos sin hembras (b2) no mudaron. Los grupos sin presencia materna (b2) mostraron $64.84 \%$ de mortalidad, mientras que con presencia materna (b1) fue de $5,35 \%$, mostrando una diferencia significativa (0.005) entre el éxito de sobrevida de las crías.

Los resultados, pese al número bajo de experimentos, muestran que la presencia de la hembra para $A$. jabaquara es imprescindible para la apertura de las ootecas, ya que sin ellas no hubo emergencia de las crías. Toyama (1999) observó en una araña solitaria, Chiracanthium japonicum (Bösenberg y Strand, 1906), Miturginae, que las crías no tienen la capacidad de romper la pared de la ooteca sin la presencia materna. En la especie solitaria, Loxosceles gaucho (Gertsch, 1967), Sicariidae, la madre realiza una abertura en la ooteca, luego golpea con los tarsos como señal de comunicación vibratoria y las crías responden con movimientos vibratorios. Este comportamiento probablemente permita la sincronización de la apertura con el grado de madurez de las crías (Japyassú et al., 2003). La participación materna en la apertura de la ootecas, como señalamos anteriormente, no es privativa de las especies con niveles de socialidad (subsocial y social), pero la apertura dependiente absolutamente de las madres, sin colaboración de las crías se registró en arañas del género Anelosimus como $A$. vierae y Anelosimus crassipes (Bösenberg y Strand, 1906), donde la apertura de la ooteca es totalmente realizada por la madre (Viera et al., 2007; Yip y Rayor, 2014). Ito y Shinkai (1993) reportaron que la madre necesita detectar el grado de maduración interno de las crías para abrir la ooteca en el momento preciso, observándose en otras especies, señales de las crías (movimientos de apéndices) dentro de la ooteca. Sin embargo, las madres no son solamente importantes en la apertura de la ooteca, sino que su presencia es muy necesaria para un mejor desarrollo de la prole, brindándoles su primera alimentación, mediante regurgitación y compartiendo presas (Viera et al., 2007). Estudios con la especie de saltícidos Psecas chapoda (Peckham y Peckham, 1894) muestran que la presencia de la hembra aumenta la sobrevida de las crías y disminuyen la tasa de mortalidad de ootecas, protegiéndolas contra enemigos naturales (Vieira y Romero, 2008). Punzo y Ludwig (2002) sugieren que el contacto precoz con la madre y hermanos es de vital importancia para el desarrollo del sistema nervioso central en 
crías de licosídos y pueden afectar también la capacidad del aprendizaje espacial, y la capacidad para capturar presas. Al retirar a la madre en Coelotes terrestris (Wider, 1834), Agelinidae, las ootecas son atacadas por hongos y ácaros (Horel y Krafft, 1986). La remoción de las madres de $C$. japonicum en los nidos disminuye la supervivencia de las ootecas y crías por presiones de depredadores (Horel y Kraft, 1986). Estos resultados apoyan la hipótesis de que una de las mayores presiones para la manutención y/o selección para el cuidado maternal es la depredación, como ha sido sugerido para otras arañas (Lubin, 1974; Fink, 1986).

Nuestros datos muestran una diferencia significativa entre la supervivencia de las crías, demostrando que la presencia de la madre es importante en la captura de presas en los primeros estadios de desarrollo y en consecuencia, para el desarrollo de las crías. Las crías sin la presencia materna parecen ser incapaces de reconocer a las presas, como fue descrito para $A$. crassipes, tienen telas más débiles y menos eficientes que las telas de las crías con sus madres (Ito y Shinkai, 1993). En Amaurobius ferox (Walckenaer, 1830), Amaurobiidae, el beneficio que los jóvenes pueden obtener a partir de la tela materna fue mayor que el uso de sus propias telas. En esta especie, la tela construida por la madre proporciona una mejor transmisión de información sobre las presas, por lo que más cantidad de crías fueron reclutadas para la captura cooperativa en un corto período de tiempo (Kim, 2005). De acuerdo a nuestro estudio, los cuidados maternos en la especie. $A$. jabaquara tienen al menos 2 funciones: ayudar a las crías a emerger de la ooteca, y capturar presas para alimentar las crías. El aprovisionamiento de presas por las madres mejora significativamente la supervivencia y el desarrollo de las crías (Yip y Rayor, 2014). Los recursos que los jóvenes reciben a través de su madre pueden tener una gran importancia en la sobrevida influyendo en su desarrollo y crecimiento, permitiendo a los jóvenes dispersarse, antes que los jóvenes que crecen sin la ayuda materna (Viera et al. 2007). La maduración más temprana les permite aumentar su éxito reproductor (Fink, 1986, Schneider, 1995, Schneider, 1996a, 1996b, Salomon y Lubin 2007; Royle, Smiseth y Kolliker, 2012; Yip y Rayor, 2014; Viera y Agnarsson, 2017; Junghanns et al, 2019).

\section{AGRADECIMIENTOS}

Agradecemos al PEDECIBA por apoyo al primer autor. A la Prefectura Municipal de Jundiaí por utilización de instalaciones de Sierra do Japi, a la Dra. Suzana Diniz por la ayuda en las colectas e identificación de los ejemplares. A los revisores anónimos que han mejorado mucho el manuscrito con sus valiosas sugerencias.

\section{BIBLIOGRAFÍA}

Agnarsson, I. (2004). Morphological phylogeny of cobweb spiders and their relatives (Araneae, Araneoidea, Theridiidae). Zoologycal Journal of the Linnean Society, 141(4), 447-626.

Agnarsson, I. (2006). A revision of the New World eximius lineage of Anelosimus (Araneae, Theridiidae) and a phylogenetic analysis using worldwide exemplars. Zoologycal Journal of the Linnean Society, 146(4), 453-593.

Aviles L. (1997). Causes and consequences of cooperation and permanent- sociality in spiders. En: J.C. Choe y B.J. Crespi (Eds.). Social Behavior in Insects and Arachnids (pp. 476-498). Cambridge Univ. Press

Avilés, L., y Guevara, J. (2017). Sociality in Spiders. En: D.R Rubenstein (Ed.). Invertebrates (pp. 188-223). Cambridge University Press.

Buskirk, R.E. (1981). Sociality in the Arachnida. En: H.R. Hermann (Ed.). Social Insects Vol. 1I (pp. 282-367). Academic Press.

Deshmukh, S.U. (2017). Suicidal maternal care in spider Stegodyphus sarasinorum, (family Eresidae). International Journal of Fauna and Biological Studies, 4(2), 114-116.

Fink, L. S. (1986). Costs and benefits of maternal behaviour in the green lynx spider (Oxyopidae, Peucetia viridans). Animal Behavior, 34, 10511060.

Foelix, R.F. (2011). Biology of Spiders. Third Edition. Oxford University Press.

Horel, A., y Krafft, B. 1986. Le comportament maternal chez lês araignées et son intervention dans lês processus sociaux. Comportament, 6, 17-29.

Ito, C., y Shinkai A. (1993). Mother-young interactions during the brood-care period in Anelosimus crassipes (Araneae:Theridiidae). Acta Arachnologica, 42, 73-81.

Kim,K. W. (2005). L'héritagematerneld'une araignée subsociale:une toile pour la prédation collective des jeunes. Comptes Rendus Biologies, 328, 89-95

Kullmann, E.J. (1972). Evolution of social behavior in spiders (Araneae; Eresidae and Theridiidae). American Zoologist, 12, 419-426.

Japyassú, H.F., Macagnan, C.R., y Knysak, I. (2003). Ooteca recognition in Loxosceles gaucho (Araneae: Sicariidae) and evolution of maternal care in spiders. Journal of Arachnology, 31, 90104.

Junghanns, A., Holm, C., Schou, M.F., Overgaard, J., Malte, H., Uhl, G., y Bilde, T. (2019). Physiological Adaptations to Extreme Maternal and Allomaternal Care in Spiders. Frontier Ecology and Evolution, 7, 305-328.

Lubin, Y. D. (1974). Adaptive advantages and the evo- 
lution of colony formation in Cytrophora (Araneae: Araneidae). Zoological Journal da Linnean Society, 54, 321-339.

Nava, A., Whitehouse, M.E.A., y Lubin, Y. (2000). Food consumption rates and competition in a communally feeding social spider, Stegodyphus dumicola (Eresidae). The Journal of Arachnology, 28, 195-200.

Norgaard, E. (1956). Environment and behaviour of Theridion saxatile. Oikos, 7, 159-192.

Punzo, F., y Ludwig, L. (2002). Contact with maternal parent and siblings affects hunting behavior,learning, and central nervous system development in spiderlings of Hogna carolinensis (Araeneae: Lycosidae). Animal Cognition, 5:63-70.

Royle, N.J., Smiseth, P.T., y Kolliker, M. (2012). The Evolution of Parental Care. Oxford University Press.

Salomon, M., y Lubin, Y. (2007). Cooperative breeding increases reproductive success in the social spider Stegodyphus dumicola (Araneae, Eresidae). Behavior Ecology Sociobiology, 61, 1743-1750.

Schneider, J.M. (1995). Survival and growth in groups of a subsocial spider (Stegodyphus lineatus). Insect Sociaux, 42, 227-248.

Schneider, J.M. (1996 $\left.{ }^{a}\right)$. Differential mortality and relative maternal investment in different life stages in Stegodyphus lineatus (Araneae, Eresidae). Journal of Arachnology, 24, 148-154.

Schneider, J.M. (1996b). Food intake, growth and relatedness in the subsocial spider Stegodyphus lineatus (Eresidae). Ethology, 102, 386-396.

Toyama, M. (1999). Adaptive advantages of maternal care and matrifagia in a foliage spider,Chiracanthium japonicum(Araneae: Clubionidae). Journal of Ethology, 17, 33-39.

Viera, C., y Agnarsson, I (2017). Parental care and sociality: En: C. Viera y M. Gonzaga (Eds.). Behaviour and Ecology of Spiders: Contribution from the Neotropical region (pp. 351-383). Springer Press.

Viera, C., Ghione, S., y Costa, F.G. (2007). Mechanisms underlying egg-sac opening in the subsocial spider Anelosimus cf. studiosus (Araneae Theridiidae). Ethology Ecology $y$ Evolution, 19, 61-67.

Vieira, C., y Romero, G.Q. (2008). Maternal care in a Neotropical jumping spider (Salticidae). Journal of Zoology, 276, 237-241.

Yip, E.C., y Rayor, L.S. (2014). Maternal care and subsocial behaviour in spiders. Biological Reviews, 89, 427-449.

Editor de Sección: Anita Aisenberg 